\title{
Introduction to the Special Issue on Face Analysis Applications
}

At the core of human social interactions is our ability to communicate information in multiple ways, implicitly and explicitly, and the human face is one of the most powerful carriers of information, about identity, gender, age, ethnicity, health, emotional state, and physical wellness, to name a few. This motivated several studies, addressed within a broad range of disciplines, with the aim to analyze the appearance, 3D structure, and dynamics of the face to extract useful information for recognition, classification, prediction, and synthesis. The definition of a computational model to formalize the precise nature of this process remains challenging due to the high-dimensional, dynamic information space required to model the appearance and deformations of the face. Nevertheless, many research teams at the crossband of computer vision, machine learning, and social sciences have investigated the use of computational models to process still images and videos, $2 \mathrm{D}$ and 3D data, to enable face recognition in the wild, face learning for re-identification, age, gender, and ethnicity estimation from face images, appearance prediction across aging, expression analysis for emotional and sentiment awareness, analysis of facial expressivity impairment for neurological disorder monitoring, and micro-expression recognition for deception detection.

This special issue includes six articles that were selected through a peer-review process among articles submitted in response to an open call for articles published in August of 2018. The first three articles address the task of face synthesis, how to represent in a compact form the geometry and appearance of faces to enable the generation of consistent predictions, considering that human perception is highly sensitive to non-realistic appearance. Combining texture and geometric information for realistic rendering is the focus of "A Unified Tensor-based Active Appearance Model." The proposed approach relies on the construction of a unified tensor model capturing all relevant face appearance variations. To achieve the unification across pose variations, a strategy for dealing with self-occluded faces is proposed that yields consistent shape and texture representations of pose-varied faces. Realistic face synthesis is also the topic of "Synthesizing Facial Photometries and Corresponding Geometries Using Generative Adversarial Networks," which describes a model to parametrize both texture and geometric information with respect to a shared 2D domain to enable the definition of a GAN loss for training a generator network to synthesize consistent textures and geometries for realistic images.

A specific class of approaches to face image synthesis targets the problem of preserving the identity traits of a person in the process of generating a face with non-neutral facial expression. This is the focus of "U-Net Conditional GANs for Photo-realistic and Identity-preserving Facial Expression Synthesis," which describes U-Net Conditional Generative Adversarial Networks that can retain characterizing traits of the input face and synthesize many facial expressions. This is achieved by pairing the generated output with conditional image of other identities for the discriminator, to encourage it to learn the distinctions between the synthesized and natural images as well as between input and other identities.

In "Efficient Face Alignment with Fast Normalization and Contour Fitting Loss," the task of efficient and robust face alignment by facial landmarks detection is addressed. The proposed solution relies on the design of a lightweight Convolutional Neural Network that is trained using

(C) 2019 Copyright held by the owner/author(s).

1551-6857/2019/10-ART85

https://doi.org/10.1145/3359624 
a contour fitting loss, which dynamically adjusts the regression target so that the network can learn more accurate semantic meanings of the contour landmarks and achieve better localization performance.

"Visual Attention Analysis and Prediction on Human Faces for Children with Autism Spectrum Disorder" presents a study on the visual attention on faces by children affected by Autism Spectrum Disorder (ASD).. The study is based on a collected dataset of 300 face images, a protocol to measure the regions of attention on the face by performing eye-tracking, and a model to fine tune a deep neural network for estimating the saliency of facial regions based on eye-tracking data computed from individuals with ASD.

Finally, a novel deep network architecture, Tensor Correlation Fusion Network, is proposed in "Features-enhanced Multi-attribute Estimation with Convolutional Tensor Correlation Fusion Network," with the aim to consider correlations in the process of facial attributes learning and improve the accuracy and robustness of attribute prediction.

Pietro Pala
University of Florence, Italy
Liming Chen
Ecole Centrale de Lyon, France
Di Huang
Beihang University, China
Xiaoming Liu
Michigan State University, USA
Stefanos Zafeiriou
Imperial College, United Kingdom
Guest Editors

